\title{
Efficacy and Safety of Ramucirumab in Asian and Non-Asian Patients with Advanced Hepatocellular Carcinoma and Elevated Alpha-Fetoprotein: Pooled Individual Data Analysis of Two Randomized Studies
}

\author{
Chia-Jui Yen $^{a}$ Masatoshi Kudo ${ }^{b}$ Ho-Yeong Lim ${ }^{c}$ Chih-Hung Hsu ${ }^{d}$ \\ Arndt Vogel ${ }^{e}$ Giovanni Brandi ${ }^{f}$ Rebecca Cheng ${ }^{g}$ loana Simona Nitu ${ }^{\text {h }}$ \\ Paolo Abada ${ }^{i}$ Yanzhi Hsuj Andrew X. Zhuk Yoon-Koo Kangl \\ a Division of Hematology and Oncology, Department of Internal Medicine, National Cheng \\ Kung University Hospital, College of Medicine, National Cheng Kung University, Tainan, \\ Taiwan; ${ }^{b}$ Department of Gastroenterology and Hepatology, Kindai University Faculty of \\ Medicine, Osaka, Japan; ' Samsung Medical Center, Sungkyunkwan University School \\ of Medicine, Seoul, South Korea; ${ }^{d}$ National Taiwan University Hospital and National \\ Taiwan University Cancer Center, Taipei, Taiwan; ${ }^{\mathrm{e} H a n n o v e r ~ M e d i c a l ~ S c h o o l, ~ H a n n o v e r, ~}$ \\ Germany; ${ }^{f}$ Oncology Unit, Department of Experimental, Diagnostic and Specialty Medicine, \\ Sant'Orsola-Malpighi Hospital, University of Bologna, Bologna, Italy; ${ }^{9}$ Eli Lilly and Company, \\ Taipei, Taiwan; hEli Lilly and Company, Bucharest, Romania; 'iEli Lilly and Company, \\ Indianapolis, IN, USA; jTG Therapeutics Inc., New York, NY, USA; kMassachusetts General \\ Hospital Cancer Center, Harvard Medical School, Boston, MA, USA; 'Asan Medical Center, \\ University of Ulsan College of Medicine, Seoul, South Korea
}

\section{Keywords}

Asian patients $\cdot$ Hepatocellular carcinoma $\cdot$ Ramucirumab

\begin{abstract}
Objective: REACH-2 and REACH were randomized, placebo-controlled, double-blind, multicenter phase 3 trials which showed survival benefits of ramucirumab treatment in patients with advanced hepatocellular carcinoma (HCC) and elevated alpha-fetoprotein (AFP). We evaluated the efficacy and safety of ramucirumab in Asian and non-Asian patients with AFP $\geq 400 \mathrm{ng} / \mathrm{mL}$ from REACH-2 and REACH. Methods: We pooled Asian and non-Asian patients from the REACH-2 and REACH trials and performed an individual patient data meta-analysis. Overall survival (OS) and progression-free survival were evaluated using the Kaplan-Meier method. Hazard ratios (HRs) were estimated with a stratified Cox regression model. Results: In the pooled REACH-2 and REACH patient population, 291 Asian patients were randomly as-
\end{abstract}


signed to receive ramucirumab $(n=168)$ or placebo $(n=123)$, and 251 non-Asian patients received ramucirumab $(n=148)$ or placebo $(n=103)$. The median OS was significantly longer in the ramucirumab arm in comparison to the placebo arm for Asian patients (8.08 vs. 4.76 months, stratified HR 0.73 [95\% Cl 0.56-0.95], $p=0.0189$ ) and non-Asian patients (7.98 vs. 5.22 months, stratified HR $0.65[95 \% \mathrm{Cl} 0.49-0.86], p=0.0028)$. The overall response rate (ORR) and disease control rate (DCR) were significantly higher in the ramucirumab arm compared to the placebo arm for Asian patients (ORR: 4.2 vs. $0.8 \%$; DCR: 53.6 vs. 33.3\%) and non-Asian patients (ORR: 6.8 vs. $1.0 \%$; DCR: 59.5 vs. $41.7 \%$ ). The most common grade $\geq 3$ treatment-emergent adverse events reported in the ramucirumab arm were hypertension (7.7\%), decreased appetite (1.2\%), and ascites (1.2\%) for Asian patients and hypertension (16.9\%), ascites (8.8\%), asthenia (4.7\%), and fatigue (5.4\%) for non-Asian patients. Discussion and Conclusion: This pooled analysis of the REACH-2/REACH trials demonstrates significant benefits, with a manageable safety profile, of ramucirumab treatment in Asian and non-Asian patients with advanced $\mathrm{HCC}$ and baseline AFP $\geq 400 \mathrm{ng} / \mathrm{mL}$.

(c) 2020 The Author(s)

Published by S. Karger AG, Basel

\section{Introduction}

Worldwide, liver cancer is the sixth most commonly diagnosed cancer and the fourth leading cause of cancer deaths. Of all primary liver cancer cases, hepatocellular carcinoma (HCC) comprises 75-85\% [1]. The incidence and mortality rate is particularly high in East and Southeast Asia. The risk factors for developing HCC vary by region. In many high-risk regions, such as China, the main risk factor for HCC is chronic infection with the hepatitis B virus, whereas hepatitis $C$ is the predominant risk factor in Japan [1]. Other common causes include cirrhosis resulting from alcohol abuse and metabolic conditions including diabetes [2, 3]. Baseline alpha-fetoprotein (AFP) levels $>400 \mathrm{ng} / \mathrm{mL}$ are associated with poor prognosis in HCC, and tumors expressing high AFP commonly demonstrate high cell proliferation, high angiogenesis, and low apoptosis [4-6].

Vascular endothelial growth factor (VEGF) and VEGF receptor 2 (VEGFR-2) signaling play an important role in angiogenesis and tumor growth [7-9]. VEGF is overexpressed in HCC, indicating that it may play a pivotal role in HCC development and progression [10-12]. Ramucirumab is a human immunoglobulin G1 monoclonal antibody targeting VEGFR-2 [13], a key mediator of VEGF-induced angiogenesis [14]. REACH was a global, randomized, phase 3 trial examining the efficacy and safety of ramucirumab monotherapy in patients with advanced HCC after first-line sorafenib treatment [15]. In an unselected population, secondline treatment with ramucirumab did not significantly improve survival in comparison to placebo-treated patients. However, a significant improvement in overall survival (OS) was observed on a prespecified analysis in the ramucirumab arm in comparison to the placebo arm in patients with baseline AFP concentrations $\geq 400 \mathrm{ng} / \mathrm{mL}$ [15].

To confirm the observed survival benefits in patients with elevated AFP, the REACH-2 trial was designed. This global, randomized, phase 3 trial demonstrated improved OS in ramucirumab-treated HCC patients with AFP levels $\geq 400 \mathrm{ng} / \mathrm{mL}$ and was the first positive phase 3 trial performed in a biomarker-selected population with advanced HCC [16].

An individual patient data meta-analysis of REACH-2 and REACH provides a larger dataset for evaluation and supports the significant and clinically meaningful benefit, with a favorable safety profile, of ramucirumab treatment in comparison to placebo for patients with HCC after first-line sorafenib therapy [17]. Ethnicity is a significant factor influencing the efficacy, tolerability, and safety of anticancer treatments $[18,19]$. There is also significant 
regional heterogeneity in the etiology, and management, of HCC. We conducted a pooled subgroup analysis to investigate the efficacy and safety of ramucirumab, in comparison to placebo, in Asian and non-Asian patients with elevated AFP from REACH-2 and REACH.

\section{Subjects and Methods}

Study Design and Participants

REACH-2 (NCT02435433) and REACH (NCT01140347) were both randomized, placebo-controlled, double-blind, multicenter phase 3 trials $[15,16]$. The study designs for REACH and REACH-2 have been previously described. For both trials, eligible patients were aged 18 years or older and diagnosed with HCC via histopathological or cytological findings, or diagnosed with cirrhosis and HCC via classical imaging characteristics. Patients were previously treated with sorafenib and discontinued treatment at least 14 days before randomization. Patients had documented disease progression during or after sorafenib therapy. For REACH-2 only, patients were eligible if they had serum AFP concentrations $\geq 400 \mathrm{ng} / \mathrm{mL}$ [16].

We performed a meta-analysis by pooling Asian patients from REACH- 2 and REACH (AFP $\geq 400 \mathrm{ng} / \mathrm{mL}$ ). The Asian population was defined based on race (Asian vs. white vs. Black or African American) and included patients from Korea, China, Hong Kong, Taiwan, and Japan. Similarly, non-Asian patients from the two studies were also pooled.

Randomization and Procedures

Patient randomization and procedures for REACH and REACH-2 have been previously described [15, 16]. In the REACH trial, patients were randomized 1:1 to receive either ramucirumab or placebo. Randomization in REACH was stratified by geographical region and cause of liver disease. As the REACH trial showed potential ramucirumab benefits in patients with increased AFP, REACH-2 used a 2:1 randomization ratio to allocate a smaller percentage of patients to placebo. Randomization in REACH-2 was stratified by geographical region, macrovascular invasion, and Eastern Cooperative Oncology Group performance status.

In both trials, patients received ramucirumab $8 \mathrm{mg} / \mathrm{kg}$ or placebo intravenously over $1 \mathrm{~h}$, every 14 days, until disease progression, unacceptable toxicity, or withdrawal of consent. All patients received best supportive care. Disease assessment and imaging studies were performed according to RECIST (version 1.1) every 6 weeks during the first 6 months and every 9 weeks thereafter.

Outcomes

The primary and secondary outcome measures for REACH-2 and REACH have been described previously $[15,16]$. In this pooled analysis of REACH-2 and REACH (AFP $\geq 400 \mathrm{ng} / \mathrm{mL}$ ), we describe OS, progressionfree survival (PFS), time to tumor progression, overall response rate (ORR), time to deterioration of FACTHepatobiliary Symptom Index-8 (FHSI-8) score, disease control rate (DCR), and safety in Asian and nonAsian patients.

\section{Statistical Analysis}

Statistical analyses were performed as previously described for REACH-2 and REACH $[15,16]$. In this analysis, Asian and non-Asian patients from the REACH- 2 and REACH (AFP $\geq 400 \mathrm{ng} / \mathrm{mL}$ ) trials were pooled and analyzed at the individual patient data level. OS and PFS for Asian and non-Asian patients were evaluated using the Kaplan-Meier method. Hazard ratios (HRs) were estimated with a Cox regression model stratified by study. All safety analyses were performed in the safety population, defined as any patient who received any dose of ramucirumab or placebo.

\section{Results}

Patients

In the pooled REACH and REACH-2 patient population, a total of 291 Asian patients received ramucirumab $(n=168)$ or placebo $(n=123)$, with a total of 251 non-Asian patients receiving ramucirumab $(n=148)$ or placebo $(n=103)$. 


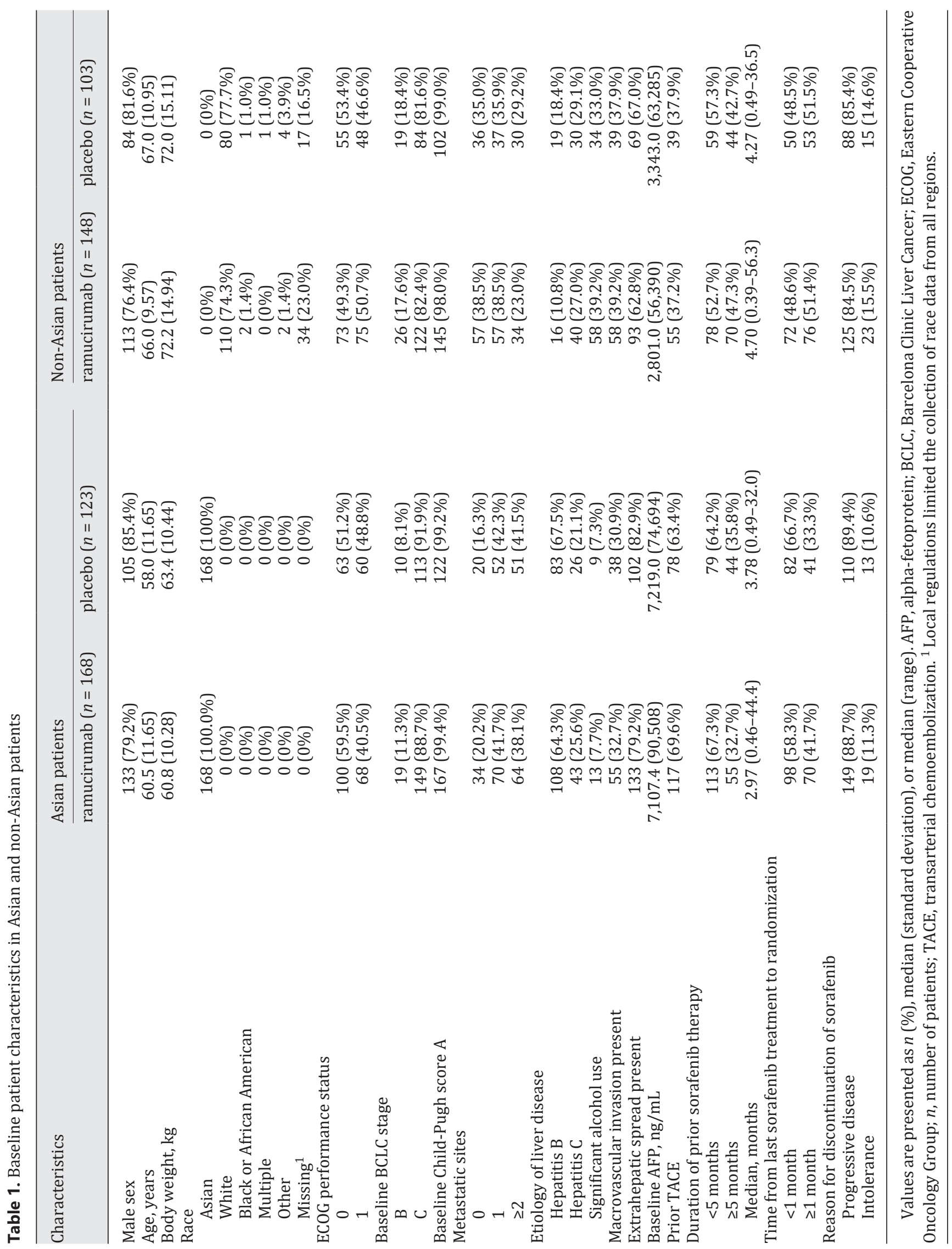




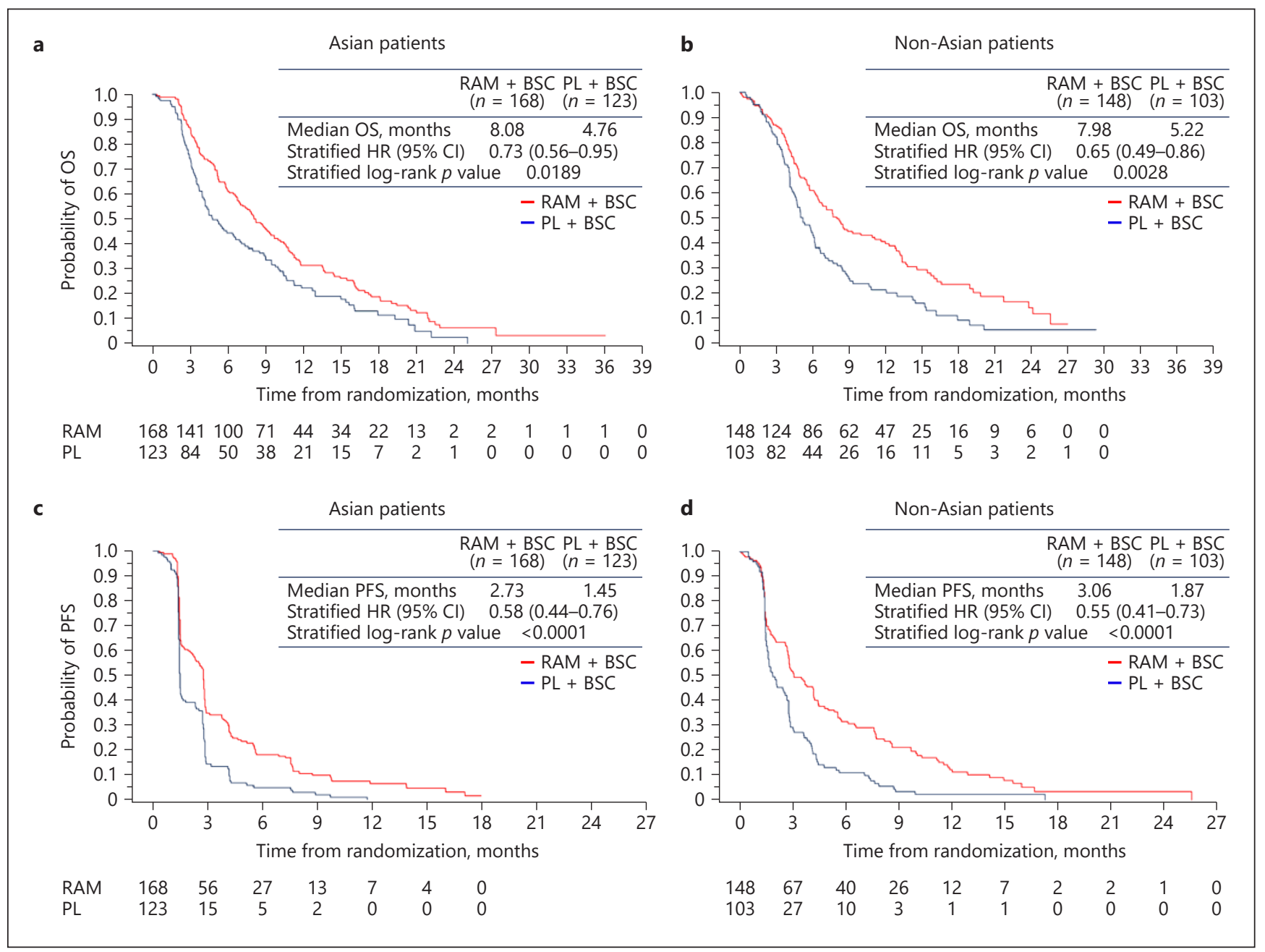

Fig. 1. Kaplan-Meier plots of OS (a, b) and PFS (c, d) for Asian (a, c) and non-Asian patients (b, d). BSC, best supportive care; $n$, number of patients; OS, overall survival; PFS, progression-free survival; PL, placebo; RAM, ramucirumab.

Baseline patient and disease characteristics for Asian and non-Asian patients are outlined in Table 1. Differences between Asian and non-Asian patients were observed for body weight (Asian patients were lighter), Barcelona Clinic Liver Cancer stage (more Asian patients were stage C), number of metastatic sites (more Asian patients exhibited $\geq 2$ sites), etiology of liver disease (more Asian patients were diagnosed with hepatitis B), significant alcohol use (smaller number of Asian patients), extrahepatic spread (greater for Asian patients), baseline AFP (higher for Asian patients), prior transarterial chemoembolization (TACE) (higher for Asian patients), and duration of prior sorafenib (Asian patients generally had shorter duration). Baseline characteristics between treatment arms within each pool of Asian and non-Asian patients were generally well balanced.

The median duration of therapy in Asian patients was 9.7 weeks (range 2-84) in the ramucirumab arm and 6.0 weeks (range 2-52) in the placebo arm. For non-Asian patients, the median duration of therapy was 12.1 weeks (range 2-107) in the ramucirumab arm and 8.1 weeks (range 2-77) in the placebo arm. Median relative dose intensity was $>98 \%$ for all patients (Asian and non-Asian). 


\begin{tabular}{|c|c|c|c|c|c|c|c|}
\hline \multicolumn{8}{|l|}{$\mathbf{a}$} \\
\hline \multicolumn{2}{|l|}{ Asian patients } & $\begin{array}{l}\text { RAM } \\
\text { group }(n / N)\end{array}$ & \multicolumn{4}{|c|}{$\begin{array}{l}\text { PL } \\
\text { group }(n / N)\end{array}$} & $\mathrm{HR}(95 \% \mathrm{Cl})$ \\
\hline \multicolumn{2}{|l|}{ Overall } & $138 / 168$ & $102 / 123$ & $1 \mapsto$ & & & $0.729(0.559-0.950)$ \\
\hline \multirow[t]{2}{*}{ Sex } & Male & $110 / 133$ & $89 / 105$ & $\mapsto \mid$ & & & $0.708(0.529-0.947)$ \\
\hline & Female & $28 / 35$ & $13 / 18$ & 1 & & & $0.898(0.439-1.873)$ \\
\hline \multirow[t]{2}{*}{ Age } & $<65$ years & $89 / 107$ & $75 / 89$ & $\mapsto$ & & & $0.724(0.526-0.995)$ \\
\hline & $\geq 65$ years & $49 / 61$ & $27 / 34$ & $\mapsto$ & -1 & & $0.733(0.446-1.203)$ \\
\hline \multirow[t]{3}{*}{ Etiology of liver disease } & Hepatitis B & $88 / 108$ & $69 / 83$ & $\mapsto \mid$ & & & $0.783(0.566-1.081)$ \\
\hline & Hepatitis C & $31 / 37$ & $20 / 23$ & 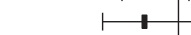 & -1 & & $0.739(0.417-1.311)$ \\
\hline & Other & $18 / 22$ & $12 / 14$ & $\longmapsto$ & & & $0.593(0.198-1.778)$ \\
\hline \multirow[t]{2}{*}{ Extrahepatic metastases } & Yes & $108 / 133$ & $85 / 102$ & 11 & & & $0.722(0.535-0.973)$ \\
\hline & No & $30 / 35$ & $17 / 21$ & $\mapsto$ & & & $0.744(0.402-1.377)$ \\
\hline \multirow[t]{2}{*}{ Macrovascular invasion } & Yes & $51 / 55$ & $33 / 38$ & $\vdash$ & -1 & & $0.907(0.580-1.417)$ \\
\hline & No & $87 / 133$ & $69 / 85$ & $\mapsto$ & & & $0.615(0.439-0.863)$ \\
\hline \multirow[t]{2}{*}{ Baseline BCLC } & B & $15 / 19$ & $8 / 10$ & 1 & -1 & & $0.611(0.247-1.511)$ \\
\hline & $\mathrm{c}$ & $123 / 149$ & $94 / 113$ & $\mapsto$ & & & $0.747(0.564-0.989)$ \\
\hline \multirow[t]{2}{*}{ Baseline ECOC PS } & 1 & $54 / 68$ & $50 / 60$ & $\mapsto$ & & & $0.721(0.479-1.087)$ \\
\hline & 0 & $84 / 100$ & $52 / 63$ & $\mapsto$ & & & $0.766(0.537-1.094)$ \\
\hline \multirow{2}{*}{ Prior locoregional therapy } & Yes & $114 / 140$ & $81 / 96$ & $\mapsto$ & & & $0.740(0.551-0.993)$ \\
\hline & No & $24 / 28$ & $21 / 27$ & 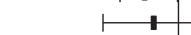 & & & $0.810(0.420-1.561)$ \\
\hline \multirow[t]{4}{*}{ Reason for sorafenib discontinuation } & Progressive disease & $124 / 149$ & $93 / 110$ & $\mapsto$ & & & $0.752(0.571-0.992)$ \\
\hline & Intolerance & $14 / 19$ & $9 / 13$ & 1 & & -1 & $0.708(0.257-1.949)$ \\
\hline & & & & $0.5 \quad 1.0$ & $0 \quad 1.5$ & $2.0 \quad 2.5$ & \\
\hline & & & & Favors RAM & & Favors PL & \\
\hline \multicolumn{8}{|l|}{ b } \\
\hline \multicolumn{2}{|l|}{ Non-Asian patients } & $\begin{array}{l}\text { RAM } \\
\text { group }(n / N)\end{array}$ & \multicolumn{3}{|c|}{$\begin{array}{l}\text { PL } \\
\text { group }(n / N)\end{array}$} & & $\mathrm{HR}(95 \% \mathrm{Cl})$ \\
\hline \multicolumn{2}{|l|}{ Overall } & $108 / 148$ & $88 / 103$ & $\longmapsto \mid$ & & & $0.647(0.486-0.863)$ \\
\hline \multirow[t]{2}{*}{ Sex } & Male & $82 / 113$ & $72 / 84$ & $\mapsto$ & & & $0.615(0.444-0.852)$ \\
\hline & Female & $26 / 35$ & $16 / 19$ & $\longmapsto \quad$ & & -1 & $0.901(0.470-1.729)$ \\
\hline \multirow[t]{2}{*}{ Age } & $<65$ years & $50 / 65$ & $35 / 44$ & $\longmapsto$ & -1 & & $0.757(0.486-1.180)$ \\
\hline & $\geq 65$ years & $58 / 83$ & $53 / 59$ & $\mapsto \mid$ & & & $0.573(0.392-0.839)$ \\
\hline Race & White & $81 / 110$ & $70 / 80$ & $\longmapsto$ & & & $0.680(0.491-0.941)$ \\
\hline & Other & $27 / 38$ & $18 / 23$ & 1 & & & $0.525(0.280-0.983)$ \\
\hline Geographical region & Region 1 & $108 / 148$ & $88 / 103$ & $\mapsto-1$ & & & $0.647(0.486-0.863)$ \\
\hline Etiology of liver disease & Hepatitis B & $12 / 16$ & $15 / 18$ & 1 & -1 & & $0.528(0.239-1.170)$ \\
\hline & Hepatitis C & $30 / 39$ & $21 / 28$ & 1 & & & $0.871(0.496-1.530)$ \\
\hline & Other & $60 / 85$ & $46 / 49$ & $\longmapsto$ & & & $0.505(0.336-0.760)$ \\
\hline Extrahepatic metastases & Yes & $69 / 93$ & $60 / 69$ & $\longmapsto$ & & & $0.640(0.449-0.912)$ \\
\hline & No & $39 / 55$ & $28 / 34$ & $\longmapsto$ & & & $0.632(0.385-1.039)$ \\
\hline Macrovascular invasion & Yes & $47 / 58$ & $33 / 39$ & 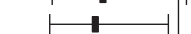 & & & $0.595(0.373-0.951)$ \\
\hline & No & $61 / 90$ & $55 / 64$ & $\longmapsto$ & & & $0.622(0.428-0.906)$ \\
\hline Baseline BCLC & B & $18 / 26$ & $16 / 19$ & $\vdash \quad$ & & & $0.444(0.214-0.921)$ \\
\hline & $\mathrm{c}$ & $90 / 122$ & $72 / 84$ & $\longmapsto$ & & & $0.669(0.488-0.918)$ \\
\hline Baseline ECOC PS & 1 & $59 / 75$ & $43 / 48$ & 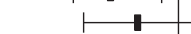 & -1 & & $0.801(0.538-1.194)$ \\
\hline & 0 & $49 / 73$ & $45 / 55$ & $\longmapsto$ & & & $0.507(0.332-0.772)$ \\
\hline Prior locoregional therapy & Yes & $58 / 76$ & $45 / 53$ & $\vdash \quad$ & & & $0.591(0.395-0.885)$ \\
\hline & No & $50 / 72$ & $43 / 50$ & $\longmapsto$ & & & $0.712(0.468-1.084)$ \\
\hline Reason for sorafenib discontinuation & Progressive disease & $90 / 125$ & $74 / 88$ & $\mapsto \mid$ & & & $0.656(0.478-0.901)$ \\
\hline & Intolerance & $18 / 23$ & $14 / 15$ & 1 & & & $0.671(0.309-1.458)$ \\
\hline & & & & $T$ & & 1 & \\
\hline & & & & 0.5 & & 2.0 & \\
\hline & & & & $\longleftarrow$ & & varc PI & \\
\hline
\end{tabular}

Fig. 2. Subgroup analysis of OS by patient and disease characteristics in Asian (a) and non-Asian patients (b). BCLC, Barcelona Clinic Liver Cancer; ECOG PS, Eastern Cooperative Oncology Group performance status; $n$, number of events; $N$, number of patients in specified category; OS, overall survival; PL, placebo; RAM, ramucirumab. 
Table 2. Overall response

\section{Asian patients}

ramucirumab

$(n=168)$

$0(0 \%)$
$7(4.2 \%)$
$83(49.4 \%)$
$71(42.3 \%)$
$7(4.2 \%)$
$7(4.2 \%)$
$90(53.6 \%)$

$0(0 \%)$
$1(0.8 \%)$
$40(32.5 \%)$
$70(56.9 \%)$
$12(9.8 \%)$
$1(0.8 \%)$
$41(33.3 \%)$

placebo $\quad p$ value

$(n=123)$

\begin{tabular}{ll} 
Non-Asian patients \\
\hline $\begin{array}{ll}\text { ramucirumab } & \text { placebo }\end{array}$ \\
$(n=148)$ & $(n=103)$
\end{tabular}

\footnotetext{
Best overall response Complete response Partial response Stable disease Progressive disease Not evaluable ORR DCR
}

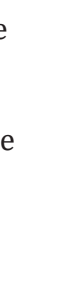

Values are presented as $n(\%)$

\section{Efficacy}

For Asian patients, median OS was significantly longer in the ramucirumab arm $(8.08$ months) in comparison to the placebo arm (4.76 months) (stratified HR 0.73 [95\% CI $0.56-$ $0.95], p=0.0189$ ). Similarly, for non-Asian patients, median OS was significantly improved in the ramucirumab arm (7.98 months) compared to the placebo arm (5.22 months) (stratified HR 0.65 [95\% CI 0.49-0.86], $p=0.0028$ ). Kaplan-Meier survival curves for OS are presented in Figure 1. Analysis of OS by patient and tumor characteristics consistently favored ramucirumab treatment in both Asian and non-Asian patients (Fig. 2).

Median PFS for Asian patients was significantly improved in the ramucirumab arm (2.73 months) compared to the placebo arm (1.45 months) (stratified HR 0.58 [95\% CI 0.44-0.76], $p<0.0001)$. For non-Asian patients, median PFS was also significantly longer in the ramucirumab arm (3.06 months) compared to the placebo arm (1.87 months) (stratified HR 0.55 [95\% CI 0.41-0.73], $p<0.0001$ ). Kaplan-Meier survival curves for PFS are presented in Figure 1.

The ORR in Asian patients was 4.2\% in the ramucirumab arm (95\% CI 1.1-7.2) and $0.8 \%$ in the placebo arm (95\% CI 0.0-2.4) ( $p=0.132)$, and the DCR was significantly higher for ramucirumab-treated patients $(53.6 \%, 95 \%$ CI $46.0-61.1)$ compared to placebo-treated patients (33.3\%, 95\% CI 25.0-41.7) ( $p=0.0043)$. For non-Asian patients, the ORR was $6.8 \%$ in the ramucirumab arm $(95 \% \mathrm{CI} 2.7-10.8)$ compared to $1.0 \%$ in the placebo arm $95 \% \mathrm{CI}$ $0.0-2.9 \%)(p=0.027)$, and the DCR was significantly higher for ramucirumab-treated patients (59.5\%, 95\% CI 51.5-67.4) compared to placebo-treated patients (41.7\%, 95\% CI 32.2-51.3) $(p=0.0067)$ (Table 2).

For Asian patients, median time to deterioration of FHSI-8 scores was 2.73 months (95\% CI 1.51-4.17) in the ramucirumab arm and 1.87 months (95\% CI 1.48-2.83) in the placebo arm (HR 0.89 [95\% CI 0.62-1.28], $p=0.510$ ). For non-Asian patients, median time to deterioration of FHSI-8 scores was 4.17 months $(95 \% \mathrm{CI} 3.02-5.52)$ in the ramucirumab arm and 1.94 months (95\% CI 1.61-2.83) in the placebo arm (HR 0.58 [95\% CI 0.40-0.85], $p=0.005$ ). Time to deterioration for individual parameters was comparable between Asian and nonAsian patients for the majority of factors (Fig. 3).

\section{Safety}

The Asian safety population included 168 patients in the ramucirumab arm and 121 in the placebo arm. For non-Asian patients, the safety population consisted of 148 patients in the ramucirumab arm and 102 patients in the placebo arm.

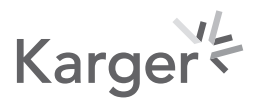


a

Asian patients

FHSI-8

Total score

Lack of energy

Nausea

Pain

Weight loss

Back pain

Fatigue

Jaundice

Discomfort in stomach

\section{b}

Non-Asian patients

FHSI-8

Total score

Lack of energy

Nausea

Pain

Weight loss

Back pain

Fatigue

Jaundice

Discomfort in stomach

$\begin{array}{ll}\text { RAM } & \mathrm{PL} \\ \text { group }(n / N) & \text { group }(n / N) \\ 85 / 168 & 53 / 123 \\ 80 / 168 & 49 / 123 \\ 59 / 168 & 37 / 123 \\ 86 / 168 & 51 / 123 \\ 52 / 168 & 37 / 123 \\ 66 / 168 & 43 / 123 \\ 84 / 168 & 48 / 123 \\ 20 / 168 & 17 / 123 \\ 96 / 168 & 43 / 123\end{array}$

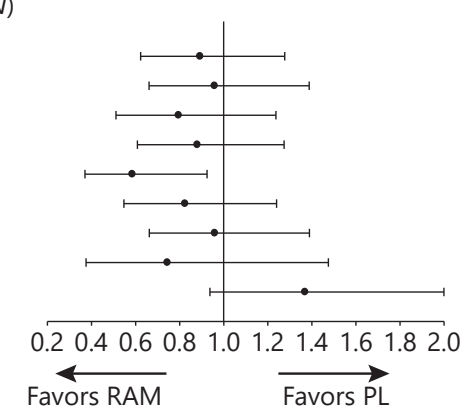

$\operatorname{HR}(95 \% \mathrm{Cl})$

$p$ value

$0.892(0.623-1.277)$

$0.958(0.661-1.388)$

$0.795(0.511-1.237)$

$0.880(0.608-1.274)$

$0.585(0.370-0.924)$

$0.824(0.547-1.240)$

$0.959(0.662-1.389)$

$0.744(0.375-1.475)$

$1.369(0.937-1.999)$

0.1018

HR $(95 \% \mathrm{Cl})$

$p$ value

group $(n / N)$

group $(n / N)$

$69 / 148$

$75 / 148$

$43 / 148$

$61 / 148$

$54 / 148$

$49 / 148$

$74 / 148$

$10 / 148$

$52 / 148$

$51 / 103$

$47 / 103$

$25 / 103$

$48 / 103$

$30 / 103$

$42 / 103$

$53 / 103$

$7 / 103$

$38 / 103$
$0.581(0.398-0.849) \quad 0.0053$

$0.935(0.645-1.356) \quad 0.7523$

$0.823(0.492-1.378) \quad 0.4537$

$0.579(0.389-0.861) \quad 0.0067$

$0.795(0.498-1.270) \quad 0.3461$

$0.496(0.321-0.767) \quad 0.0014$

$0.669(0.464-0.965) \quad 0.0320$

$0.684(0.253-1.848) \quad 0.4532$

$0.705(0.456-1.089) \quad 0.1166$

Fig. 3. Time to deterioration of patient-reported symptoms using the FHSI-8 in Asian (a) and non-Asian (b) patients. FHSI-8, FACT-Hepatobiliary Symptom Index-8; $n$, number of events; $N$, number of patients in intention to treat population (also number in category); PL, placebo; RAM, ramucirumab.

Overall, the incidence of all grade and grade $\geq 3$ treatment-emergent adverse events (TEAEs) was higher in the ramucirumab arm compared to the placebo arm in both Asian and non-Asian patients. Any grade and grade $\geq 3$ TEAEs reported in $\geq 15 \%$ of patients in the ramucirumab arm of Asian and non-Asian patients are shown in Table 3. For Asian patients, any grade TEAEs occurring in $\geq 15 \%$ of patients and at a higher rate in the ramucirumab arm (at least $10 \%$ difference) compared to the placebo arm were peripheral edema, proteinuria, and hypertension. The most common grade $\geq 3$ TEAEs reported in the ramucirumab arm were hypertension (7.7\%), decreased appetite (1.2\%), and ascites (1.2\%). For non-Asian patients, any grade TEAEs occurring in $\geq 15 \%$ patients and at a higher rate in the ramucirumab arm (at least $10 \%$ difference) compared to the placebo arm were peripheral edema, proteinuria, hypertension, asthenia, and headache. The most common grade $\geq 3$ TEAEs reported in the ramucirumab arm were hypertension (16.9\%), ascites (8.8\%), asthenia (4.7\%), and fatigue (5.4\%). Peripheral edema was the most common TEAE (any grade), while hypertension was the most common grade $\geq 3$ TEAE in the ramucirumab arm for both Asian and non-Asian patients.

The incidence of adverse events of special interest (AESI) occurring in Asian and nonAsian patients is shown in Table 3. Common AESI of any grade in the ramucirumab arm of both Asian and non-Asian patients were epistaxis, proteinuria, and hypertension. The frequency of high-grade AESI was similar between Asian and non-Asian patients. For Asian patients, serious adverse events were reported in $31.5 \%$ of patients in the ramucirumab arm 
Table 3. Adverse events

Ramucirumab

any grade grade $\geq 3$

\section{Placebo}

any grade

grade $\geq 3$

\section{TEAEs in $\geq 15 \%$ in the ramucirumab arm}

\begin{tabular}{|c|c|c|c|c|}
\hline Asian patients & \multicolumn{2}{|c|}{$n=168$} & \multicolumn{2}{|c|}{$n=121$} \\
\hline Any & $159(94.6 \%)$ & $80(47.6 \%)$ & $110(90.9 \%)$ & $58(47.9 \%)$ \\
\hline Peripheral edema & $42(25.0 \%)$ & $0(0 \%)$ & $17(14.0 \%)$ & $0(0 \%)$ \\
\hline Proteinuria & $34(20.2 \%)$ & $1(0.6 \%)$ & $9(7.4 \%)$ & $0(0 \%)$ \\
\hline Decreased appetite & $33(19.6 \%)$ & $2(1.2 \%)$ & $27(22.3 \%)$ & $0(0 \%)$ \\
\hline Hypertension & $31(18.5 \%)$ & $13(7.7 \%)$ & $8(6.6 \%)$ & $3(2.5 \%)$ \\
\hline Fatigue & $30(17.9 \%)$ & $0(0 \%)$ & $14(11.6 \%)$ & $2(1.7 \%)$ \\
\hline Ascites & $28(16.7 \%)$ & $2(1.2 \%)$ & $12(9.9 \%)$ & $4(3.3 \%)$ \\
\hline Non-Asian patients & \multicolumn{2}{|c|}{$n=148$} & \multicolumn{2}{|c|}{$n=102$} \\
\hline Any & 147 (99.3\%) & $101(68.2 \%)$ & $96(94.1 \%)$ & $58(56.9 \%)$ \\
\hline Peripheral edema & $50(33.8 \%)$ & $3(2.0 \%)$ & $21(20.6 \%)$ & $0(0 \%)$ \\
\hline Fatigue & $46(31.1 \%)$ & $8(5.4 \%)$ & $25(24.5 \%)$ & $4(3.9 \%)$ \\
\hline Nausea & $39(26.4 \%)$ & $0(0 \%)$ & $18(17.6 \%)$ & $0(0 \%)$ \\
\hline Ascites & $38(25.7 \%)$ & $13(8.8 \%)$ & $21(20.6 \%)$ & $5(4.9 \%)$ \\
\hline Abdominal pain & $38(25.7 \%)$ & $3(2.0 \%)$ & $25(24.5 \%)$ & $4(3.9 \%)$ \\
\hline Decreased appetite & $37(25.0 \%)$ & $2(1.4 \%)$ & $19(18.6 \%)$ & $1(1.0 \%)$ \\
\hline Hypertension & $35(23.6 \%)$ & $25(16.9 \%)$ & $12(11.8 \%)$ & $5(4.9 \%)$ \\
\hline Asthenia & $34(23.0 \%)$ & $7(4.7 \%)$ & $13(12.7 \%)$ & $1(1.0 \%)$ \\
\hline Diarrhea & $33(22.3 \%)$ & $1(0.7 \%)$ & $17(16.7 \%)$ & $1(1.0 \%)$ \\
\hline Headache & $30(20.3 \%)$ & $0(0 \%)$ & $8(7.8 \%)$ & $0(0 \%)$ \\
\hline Proteinuria & $22(14.9 \%)$ & $3(2.0 \%)$ & $3(2.9 \%)$ & $0(0 \%)$ \\
\hline \multicolumn{5}{|l|}{ AESI } \\
\hline Asian patients & \multicolumn{2}{|c|}{$n=168$} & \multicolumn{2}{|c|}{$n=121$} \\
\hline Liver injury or failure $^{1}$ & $63(37.5 \%)$ & $26(15.5 \%)$ & $38(31.4 \%)$ & $28(23.1 \%)$ \\
\hline Bleeding/hemorrhage ${ }^{1}$ & $45(26.8 \%)$ & $7(4.2 \%)$ & $20(16.5 \%)$ & $7(5.8 \%)$ \\
\hline Gastrointestinal hemorrhage events ${ }^{1}$ & $12(7.1 \%)$ & $5(3.0 \%)$ & $11(9.1 \%)$ & $5(4.1 \%)$ \\
\hline Infusion-related reaction & $6(3.6 \%)$ & $0(0 \%)$ & $0(0 \%)$ & $0(0 \%)$ \\
\hline Venous thromboembolic events ${ }^{1}$ & $2(1.2 \%)$ & $0(0 \%)$ & $2(1.7 \%)$ & $1(0.8 \%)$ \\
\hline Arterial thromboembolic events ${ }^{1}$ & $1(0.6 \%)$ & $0(0 \%)$ & $1(0.8 \%)$ & $1(0.8 \%)$ \\
\hline Wound hemorrhage & $1(0.6 \%)$ & $0(0 \%)$ & $0(0 \%)$ & $0(0 \%)$ \\
\hline Non-Asian patients & \multicolumn{2}{|c|}{$n=148$} & \multicolumn{2}{|c|}{$n=102$} \\
\hline Liver injury or failure $^{1}$ & $77(52.0 \%)$ & $37(25.0 \%)$ & $49(48.0 \%)$ & $31(30.4 \%)$ \\
\hline Bleeding/hemorrhage ${ }^{1}$ & $34(23.0 \%)$ & $8(5.4 \%)$ & $20(19.6 \%)$ & $8(7.8 \%)$ \\
\hline Gastrointestinal hemorrhage events ${ }^{1}$ & $12(8.1 \%)$ & $6(4.1 \%)$ & $8(7.8 \%)$ & $7(6.9 \%)$ \\
\hline Arterial thromboembolic events ${ }^{1}$ & $4(2.7 \%)$ & $3(2.0 \%)$ & $2(2.0 \%)$ & $1(1.0 \%)$ \\
\hline Infusion-related reaction & $4(2.7 \%)$ & $1(0.7 \%)$ & $1(1.0 \%)$ & $0(0 \%)$ \\
\hline Venous thromboembolic events ${ }^{1}$ & $1(0.7 \%)$ & $1(0.7 \%)$ & $4(3.9 \%)$ & $4(3.9 \%)$ \\
\hline Wound hemorrhage & $1(0.7 \%)$ & $0(0 \%)$ & $0(0 \%)$ & $0(0 \%)$ \\
\hline
\end{tabular}

Values are presented as $n(\%)$. AESI, adverse events of special interest; $n$, number of patients; TEAEs, treatment-emergent adverse events. ${ }^{1}$ Pooled adverse event terms.

and in $29.8 \%$ of patients in the placebo arm; 7.7 and $6.6 \%$, respectively, were related to the study treatment. Similarly, for non-Asian patients, serious adverse events were reported in $39.9 \%$ in the ramucirumab arm and in $38.2 \%$ in the placebo arm; 12.8 and $8.8 \%$, respectively, were related to the study treatment. In terms of study discontinuation due to any adverse event, $12.5 \%$ of Asian patients in the ramucirumab arm and $7.4 \%$ of patients in the placebo arm discontinued, with 7.7 and 3.3\%, respectively, considered related to the study treatment. 
For non-Asian patients, $20.9 \%$ of patients in the ramucirumab arm and $13.7 \%$ of patients in the placebo arm discontinued study treatment due to an adverse event, with 11.5 and 3.9\%, respectively, considered related to the study treatment.

For Asian patients, deaths due to an adverse event occurred in 1 patient in each treatment arm, considered to be not related to the study treatment. For non-Asian patients, deaths due to an adverse event occurred in $4.7 \%$ of patients in the ramucirumab arm, with $2.7 \%$ considered to be due to the study treatment; $2.9 \%$ of non-Asian patients in the placebo arm died, but no deaths were considered to be related to the study treatment.

\section{Discussion and Conclusion}

HCC is a heterogeneous disease, with treatment and prognosis dependent on many factors including cancer stage, underlying liver disease, and AFP levels [4, 20]. In addition, treatment approaches vary by region, further adding to heterogeneity in patient outcomes [21]. Sorafenib was the first drug to show improved median OS in patients with HCC and is widely used as a first-line treatment [22]. Significant survival benefits are observed in patients with advanced HCC who are treated with sorafenib [22]; however, the median OS in Asian patients is shorter compared to that in Western patients [22, 23]. In the present analysis, treatment with sorafenib was shorter in Asian patients in comparison to non-Asian patients. The shorter OS outcome in Asian compared to Western patients may be associated with differences in baseline characteristics, such as tumor burden or hepatitis infection, or regional differences in disease management [21, 24, 25]. Furthermore, sorafenib treatment is often accompanied by toxicities in all regions [23]. The overall incidence of drug-related adverse events in Asian patients treated with sorafenib was $81.9 \%$, the most commonly reported events being hand and foot skin reactions, diarrhea, fatigue, rash, and hypertension [23]. Therefore, second-line treatments are needed for patients who progress after sorafenib therapy or are unable to continue with sorafenib due to toxicity.

This pooled subgroup analysis of the global, randomized, phase 3 REACH-2/REACH trials demonstrates statistically significant and consistent survival benefits of ramucirumab treatment in Asian and non-Asian patients with advanced HCC and baseline AFP $\geq 400 \mathrm{ng} /$ $\mathrm{mL}$. Time to deterioration of patient-reported symptoms favored ramucirumab treatment in both Asian and non-Asian patients. Despite differences in some baseline patient and disease characteristics between Asian and non-Asian patients, the survival benefit was consistent and robust across all subgroups. Some of these baseline differences were expected and related to ethnic/regional variances such as body weight, TACE utilization, and hepatitis B incidence. Notably, in Asian patients the benefits of ramucirumab are consistent regardless of hepatitis $\mathrm{B}$ or $\mathrm{C}$ etiology. Many of the differences observed in Asian compared to non-Asian patients are commonly associated with a poorer prognosis, such as increased number of metastatic sites, increased extrahepatic spread, and a higher proportion of patients at Barcelona Clinic Liver Cancer stage $\mathrm{C}$.

Despite the poor prognosis of patients from the Asia-Pacific region, a similar treatment effect was observed in patients with HCC treated with sorafenib. HRs were comparable in patients from the Asia-Pacific region in comparison to the population from the SHARP trial, where patients were predominantly recruited from Europe and Australasia (HR 0.68 vs. 0.69) $[22,23]$. However, the overall prognosis of patients from the Asia-Pacific trial differed from those of the SHARP trial, with shorter median OS for patients in the Asia-Pacific region (AsiaPacific, 6.5 months [sorafenib] vs. 4.2 months [placebo]; SHARP, 10.7 months [sorafenib] vs. 7.9 months [placebo]). Conversely, we demonstrated that the prognosis and treatment benefit are comparable for Asian and non-Asian patients in terms of median OS (Asian, 8.08 months

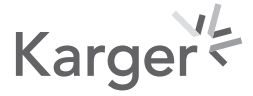


[ramucirumab] vs. 4.76 months [placebo]; non-Asian, 7.98 months [ramucirumab] vs. 5.22 months [placebo]), and in alignment with the pooled analyses of total patients from REACH-2/ REACH with baseline AFP $\geq 400 \mathrm{ng} / \mathrm{mL}$ (8.1 vs. 5.0 months). Overall, in terms of median OS and PFS, we did not observe any significant regional differences.

High AFP concentrations $(\geq 400 \mathrm{ng} / \mathrm{mL})$ are associated with aggressive disease $[5,15$, 26-29]. All patients in this analysis had an AFP concentration $\geq 400 \mathrm{ng} / \mathrm{mL}$, but the East Asian population exhibited even higher AFP concentrations in comparison to non-East Asian patients. Despite higher AFP concentrations in the East Asian population, the prognosis and survival benefit of ramucirumab treatment was notably consistent between both East Asian and nonAsian patients. It has been suggested that tumors expressing AFP may represent a biologically distinct subtype of HCC associated with poor prognosis, more stem-cell-like features (such as epithelial cell adhesion molecule expression) [30,31], increased VEGF pathway activity, and increased activity of VEGFR-2-targeted antibodies in preclinical models [32]. It is possible that the prognostic impact of AFP is lessened once AFP reaches significantly high levels where tumor behavior may become more homogeneous, and as a consequence the impact of differences in AFP observed between the East Asian and non-Asian populations are less apparent. However, as mentioned above, there are also well-known geographical differences in disease characteristics and management that may also have influenced the outcomes of patients. For example, hepatitis B is more prevalent in Asia and is also commonly associated with elevated serum AFP levels [33, 34], and some have suggested that it may impact disease prognosis.

Overall, the survival benefits of ramucirumab were comparable between Asian and nonAsian patients. ORR and DCR were also comparable, with statistically significant benefits observed in both Asian and non-Asian patients treated with ramucirumab. Improvements in OS and PFS observed in this pooled analysis of Asian and non-Asian patients are comparable to the findings reported in REACH-2 [16] and in the pooled analysis of REACH-2/REACH [17]. In the East Asian subgroup analysis of the REACH trial, ramucirumab did not significantly prolong OS [35]. However, in patients with baseline AFP $\geq 400 \mathrm{ng} / \mathrm{mL}$, a potentially larger survival benefit was observed for both East Asian and non-East Asian patients [35]. In this East Asian analysis from REACH, a lack of significance in terms of OS improvement [35] may have been attributed to the relatively small number of East Asian patients. With a larger cohort of Asian patients in the current REACH-2/REACH analysis, survival benefits now reach significance.

The efficacy benefits observed for Asian and non-Asian patients were achieved with a manageable safety profile. The majority of TEAEs and AESI in Asian patients were grade 3 or below, and the grade $\geq 3$ TEAEs and AESI were generally comparable between Asian and nonAsian patients. The most common grade $\geq 3$ TEAE in both Asian and non-Asian patients was hypertension. Liver injury and bleeding events are of particular concern in patients with HCC. Grade $\geq 3$ bleeding/hemorrhage events were low, and comparable, between patients in the ramucirumab and placebo arms. The most common TEAE (any grade and grade $\geq 3$ ) in both Asian and non-Asian patients was ascites. Importantly, there was no increase in high-grade AESI in Asian compared to non-Asian patients - this despite the prevalence of poor prognostic factors among Asian patients, potentially placing these patients at higher risk of developing adverse events following ramucirumab treatment, as well as the increased use of TACE in Asian patients, which may increase the risk of liver events. The overall observed safety profiles were consistent with those reported in other ramucirumab trials [16, 17, 36-39]. Note that for the REACH-2 trial, patients with an intolerance to sorafenib were enrolled. We demonstrated that these patients are able to tolerate ramucirumab treatment while maintaining quality of life.

A limitation of the REACH trials is that a biological link/mechanism of action between elevated AFP and ramucirumab has not been established. Interestingly, post hoc analysis of 
the REACH trial demonstrated that treatment with ramucirumab prolonged time to AFP progression, slowed AFP increase, and was more likely to induce AFP response [40]. AFP expression and its relation to poor outcomes and aggressive tumor features is not well understood and warrants further investigation. Potentially, ramucirumab is more effective in certain HCC subtypes, and it has been suggested that AFP expression may define novel prognostic subtypes of HCC [30]. Indeed, a recent HCC biomarker study demonstrated that tumors with high AFP expression show significant activation of VEGF signaling, which provides further evidence of a mechanism for ramucirumab benefit in HCC patients with elevated AFP [41]. Potential heterogeneity between the REACH-2 and REACH trials in terms of previous therapy and patient characteristics should be noted, although no heterogeneity in treatment effect was observed in REACH-2 [16]. We report a post hoc subset analysis, and therefore caution should be used when interpreting the results. Despite this limitation, the efficacy observations in Asian and non-Asian patients with AFP $\geq 400 \mathrm{ng} / \mathrm{mL}$ were consistent and therefore seem unlikely to be due to chance. Further studies focusing on the relationships between AFP concentration, prognosis, and treatment efficacy in HCC are warranted.

Ramucirumab is currently indicated for advanced/recurrent gastric cancer, colorectal cancer, and non-small-cell lung cancer and is used in routine clinical practice. Recently, based on the REACH-2 trial, ramucirumab was approved by the Food and Drug Administration and the European Medicines Agency as a single agent for HCC in patients with AFP $\geq 400 \mathrm{ng} / \mathrm{mL}$ and previous treatment with sorafenib. Similarly, in Asia, ramucirumab was recently approved by the Pharmaceutical and Medical Devices Agency and by the Taiwan Food and Drug Administration. Based on the findings of this subanalysis, we believe ramucirumab is likely to be beneficial in Asian patients with advanced HCC and elevated AFP level who are intolerant to, or have progressed on, sorafenib - a population associated with poor prognosis and aggressive disease.

\section{Acknowledgments}

The authors thank the patients and their families/caregivers, the study investigators and their staff, the independent data monitoring committee, and the REACH-2 and REACH clinical trial teams. Project management was provided by Esther Gonzalez of Eli Lilly and Company. Medical writing assistance was provided by Lisa Cossens and editorial assistance by Rod Everhart and Antonia Baldo from Syneos Health, and funded by Eli Lilly and Company.

\section{Statement of Ethics}

Both REACH and REACH-2 followed the principles of the Declaration of Helsinki and the Good Clinical Practice Guidelines of the International Conference on Harmonisation. Each center's institutional review board or ethics committee approved the study, and all patients provided written informed consent.

\section{Disclosure Statement}

M. Kudo has had a consulting/advisory role with Bayer, Bristol-Myers Squibb, Eisai Co., Ltd., and MSD; has received honoraria from AbbVie Inc., Bayer, Bristol-Myers Squibb, EA Pharma Co., Ltd., Eisai Co., Ltd., Gilead Sciences Inc., Merck Serono, MSD, Novartis, Pfizer, and Taiho Pharmaceutical; and has received research funding from AbbVie Inc., Astellas Pharma Inc., Bristol-Myers Squibb, Chugai Pharmaceutical Co., Ltd., Daiichi Sankyo Co., Ltd., Otsuka Pharmaceutical Co., Ltd., and Taiho Pharmaceutical. H.-Y. Lim is a member of the steering committee of Bayer. He participated in advisory board meetings for Bayer, Ipsen, Eisai, and Ono. C.-H. Hsu declares advisory board membership for Bristol-Myers Squibb, Ono, Merck/Serono, and Roche/Genentech, and research funding from MSD. A. Vogel declares honoraria from Lilly, Bayer, MSD, 
Roche, Novartis, AstraZeneca, and Beigene. R. Cheng, I.S. Nitu, and P. Abada are employees and stock holders at Eli Lilly and Company. Y. Hsu is a former employee of Eli Lilly and Company and declares stock ownership and a pending patent at Eli Lilly and Company. A.X. Zhu has had a consulting/advisory role with AstraZeneca Pharmaceuticals, Bayer, Bristol-Myers Squibb, Eisai Co., Ltd., Eli Lilly, Exelixis, Merck, Novartis, and Sanofi; and has received honoraria from Eli Lilly, Bayer, Bristol-Myers Squibb, Merck, and Novartis. Y.-K. Kang is a consultant for Eli Lilly and Company, BMS, Ono, Bayer, and Blueprint. All other authors report no conflicts of interest.

\section{Funding Sources}

This study was funded by Eli Lilly and Company. Medical writing assistance was provided by Syneos Health and funded by Eli Lilly and Company.

\section{Author Contributions}

P. Abada and A.X. Zhu were involved in study conception and design. C.-J. Yen, M. Kudo, H.-Y. Lim, A. Vogel, G. Brandi, P. Abada, and A.X. Zhu were involved in patient recruitment. M. Kudo, H.-Y. Lim, C.-H. Hsu, A. Vogel, G. Brandi, A.X. Zhu, and Y.-K. Kang were involved in data collection. All authors were involved in data review and analysis as well as drafting and critical review of the manuscript and provided final approval for submission.

\section{References}

1 Bray F, Ferlay J, Soerjomataram I, Siegel RL, Torre LA, Jemal A. Global cancer statistics 2018: GLOBOCAN estimates of incidence and mortality worldwide for 36 cancers in 185 countries. CA Cancer J Clin. 2018 Nov;68(6): 394-424.

2 Gomaa AI, Khan SA, Toledano MB, Waked I, Taylor-Robinson SD. Hepatocellular carcinoma: epidemiology, risk factors and pathogenesis. World J Gastroenterol. 2008 Jul;14(27):4300-8.

3 Davila JA, Morgan RO, Shaib Y, McGlynn KA, El-Serag HB. Diabetes increases the risk of hepatocellular carcinoma in the United States: a population based case control study. Gut. 2005 Apr;54(4):533-9.

4 Gomaa AI, Khan SA, Leen EL, Waked I, Taylor-Robinson SD. Diagnosis of hepatocellular carcinoma. World J Gastroenterol. 2009 Mar;15(11):1301-14.

5 Nakazawa T, Hidaka H, Takada J, Okuwaki Y, Tanaka Y, Watanabe M, et al. Early increase in $\alpha$-fetoprotein for predicting unfavorable clinical outcomes in patients with advanced hepatocellular carcinoma treated with sorafenib. Eur J Gastroenterol Hepatol. 2013 Jun;25(6):683-9.

6 Mitsuhashi N, Kobayashi S, Doki T, Kimura F, Shimizu H, Yoshidome H, et al. Clinical significance of alphafetoprotein: involvement in proliferation, angiogenesis, and apoptosis of hepatocellular carcinoma. J Gastroenterol Hepatol. 2008 Jul;23(7 Pt 2):e189-97.

7 Sullivan LA, Brekken RA. The VEGF family in cancer and antibody-based strategies for their inhibition. MAbs. 2010 Mar-Apr;2(2):165-75.

8 Amini A, Masoumi Moghaddam S, Morris DL, Pourgholami MH. The critical role of vascular endothelial growth factor in tumor angiogenesis. Curr Cancer Drug Targets. 2012 Jan;12(1):23-43.

9 Tugues S, Koch S, Gualandi L, Li X, Claesson-Welsh L. Vascular endothelial growth factors and receptors: antiangiogenic therapy in the treatment of cancer. Mol Aspects Med. 2011 Apr;32(2):88-111.

10 Mao CS, Yin H, Ning HB, Peng Z, Li K, Ding GQ. Levels of HBx, VEGF, and CEACAM1 in HBV-related hepatocellular carcinoma and their correlation with cancer prognosis. Eur Rev Med Pharmacol Sci. 2017 Oct;21(17): 3827-33.

11 Zhu AX, Finn RS, Mulcahy M, Gurtler J, Sun W, Schwartz JD, et al. A phase II and biomarker study of ramucirumab, a human monoclonal antibody targeting the VEGF receptor-2, as first-line monotherapy in patients with advanced hepatocellular cancer. Clin Cancer Res. 2013 Dec;19(23):6614-23.

12 Cao G, Li X, Qin C, Li J. Prognostic Value of VEGF in Hepatocellular Carcinoma Patients Treated with Sorafenib: A Meta-Analysis. Med Sci Monit. 2015 Oct;21:3144-51.

13 Spratlin JL, Cohen RB, Eadens M, Gore L, Camidge DR, Diab S, et al. Phase I pharmacologic and biologic study of ramucirumab (IMC-1121B), a fully human immunoglobulin G1 monoclonal antibody targeting the vascular endothelial growth factor receptor-2. J Clin Oncol. 2010 Feb;28(5):780-7.

14 Tian S, Quan H, Xie C, Guo H, Lü F, Xu Y, et al. YN968D1 is a novel and selective inhibitor of vascular endothelial growth factor receptor-2 tyrosine kinase with potent activity in vitro and in vivo. Cancer Sci. 2011 Jul;102(7): 1374-80. 


\begin{tabular}{|c|c|}
\hline \multicolumn{2}{|l|}{ Liver Cancer 2020;9:440-454 } \\
\hline DOI: $10.1159 / 000506946$ & $\begin{array}{l}\text { (c) } 2020 \text { The Author(s). Published by S. Karger AG, Basel } \\
\text { www.karger.com/lic }\end{array}$ \\
\hline
\end{tabular}

Yen et al.: Efficacy of Ramucirumab in Asian HCC Patients with Elevated AFP

15 Zhu AX, Park JO, Ryoo BY, Yen CJ, Poon R, Pastorelli D, et al.; REACH Trial Investigators. Ramucirumab versus placebo as second-line treatment in patients with advanced hepatocellular carcinoma following first-line therapy with sorafenib (REACH): a randomised, double-blind, multicentre, phase 3 trial. Lancet Oncol. 2015 Jul;16(7):859-70.

16 Zhu AX, Kang YK, Yen CJ, Finn RS, Galle PR, Llovet JM, et al.; REACH-2 study investigators. Ramucirumab after sorafenib in patients with advanced hepatocellular carcinoma and increased $\alpha$-fetoprotein concentrations (REACH-2): a randomised, double-blind, placebo-controlled, phase 3 trial. Lancet Oncol. 2019 Feb;20(2):28296.

17 Zhu A, Finn R, Galle P, Llovet J, Blanc JF, Okusaka T, et al. Ramucirumab as second-line treatment in patients with advanced hepatocellular carcinoma (HCC) and elevated alpha-fetoprotein (AFP) following first-line sorafenib: pooled efficacy and safety across two global randomized Phase 3 studies (REACH-2 and REACH). Ann Oncol. 2018 Jun;29(Suppl 5):v122.

18 Rich NE, Hester C, Odewole M, Murphy CC, Parikh ND, Marrero JA, et al. Racial and ethnic differences in presentation and outcomes of hepatocellular carcinoma. Clin Gastroenterol Hepatol. 2019 Feb;17(3):551-9.e1.

19 Stewart SL, Kwong SL, Bowlus CL, Nguyen TT, Maxwell AE, Bastani R, et al. Racial/ethnic disparities in hepatocellular carcinoma treatment and survival in California, 1988-2012. World J Gastroenterol. 2016 Oct; 22(38):8584-95.

20 Grizzi F, Franceschini B, Hamrick C, Frezza EE, Cobos E, Chiriva-Internati M. Usefulness of cancer-testis antigens as biomarkers for the diagnosis and treatment of hepatocellular carcinoma. J Transl Med. 2007 Jan; 5(1):3.

21 Park JW, Chen M, Colombo M, Roberts LR, Schwartz M, Chen PJ, et al. Global patterns of hepatocellular carcinoma management from diagnosis to death: the BRIDGE Study. Liver Int. 2015 Sep;35(9):2155-66.

22 Llovet JM, Ricci S, Mazzaferro V, Hilgard P, Gane E, Blanc JF, et al.; SHARP Investigators Study Group. Sorafenib in advanced hepatocellular carcinoma. N Engl J Med. 2008 Jul;359(4):378-90.

23 Cheng AL, Kang YK, Chen Z, Tsao CJ, Qin S, Kim JS, et al. Efficacy and safety of sorafenib in patients in the AsiaPacific region with advanced hepatocellular carcinoma: a phase III randomised, double-blind, placebocontrolled trial. Lancet Oncol. 2009 Jan;10(1):25-34.

24 Peixoto RD, Renouf DJ, Gill S, Cheung WY, Lim HJ. Relationship of ethnicity and overall survival in patients treated with sorafenib for advanced hepatocellular carcinoma. J Gastrointest Oncol. 2014 Aug;5(4):259-64.

25 Abou-Alfa GK, Venook AP. The antiangiogenic ceiling in hepatocellular carcinoma: does it exist and has it been reached? Lancet Oncol. 2013 Jun;14(7):e283-8.

26 Hsu CY, Liu PH, Lee YH, Hsia CY, Huang YH, Lin HC, et al. Using serum $\alpha$-fetoprotein for prognostic prediction in patients with hepatocellular carcinoma: what is the most optimal cutoff? PLoS One. 2015 Mar; 10(3):e0118825.

27 A new prognostic system for hepatocellular carcinoma: a retrospective study of 435 patients: the Cancer of the Liver Italian Program (CLIP) investigators. Hepatology. 1998 Sep;28(3):751-5.

28 Leung TW, Tang AM, Zee B, Lau WY, Lai PB, Leung KL, et al. Construction of the Chinese University Prognostic Index for hepatocellular carcinoma and comparison with the TNM staging system, the Okuda staging system, and the Cancer of the Liver Italian Program staging system: a study based on 926 patients. Cancer. 2002 Mar; 94(6):1760-9.

29 Borzio M, Dionigi E, Rossini A, Marignani M, Sacco R, De Sio I, et al. External validation of the ITA.LI.CA prognostic system for patients with hepatocellular carcinoma: A multicenter cohort study. Hepatology. 2018 Jun; 67(6):2215-25.

30 Yamashita T, Forgues M, Wang W, Kim JW, Ye Q, Jia H, et al. EpCAM and alpha-fetoprotein expression defines novel prognostic subtypes of hepatocellular carcinoma. Cancer Res. 2008 Mar;68(5):1451-61.

31 Shan YF, Huang YL, Xie YK, Tan YH, Chen BC, Zhou MT, et al. Angiogenesis and clinicopathologic characteristics in different hepatocellular carcinoma subtypes defined by EpCAM and $\alpha$-fetoprotein expression status. Med Oncol. 2011 Dec;28(4):1012-6.

32 Lee L, Huber L, Stewart J, Mathews M, Falcon B, Chintharlapalli S. Evaluation of AFP expression as a predictive marker for response to anti-VEGFR-2 inhibition. Ann Oncol. 2017 Jun;28(Suppl 3):iii19-20.

33 Liu C, Xiao GQ, Yan LN, Li B, Jiang L, Wen TF, et al. Value of $\alpha$-fetoprotein in association with clinicopathological features of hepatocellular carcinoma. World J Gastroenterol. 2013 Mar;19(11):1811-9.

34 Yao M, Zhao J, Lu F. Alpha-fetoprotein still is a valuable diagnostic and prognosis predicting biomarker in hepatitis B virus infection-related hepatocellular carcinoma. Oncotarget. 2016 Jan;7(4):3702-8.

35 Park JO, Ryoo BY, Yen CJ, Kudo M, Yang L, Abada PB, et al. Second-line ramucirumab therapy for advanced hepatocellular carcinoma (REACH): an East Asian and non-East Asian subgroup analysis. Oncotarget. 2016 Nov; 7(46):75482-91.

36 Fuchs CS, Tomasek J, Yong CJ, Dumitru F, Passalacqua R, Goswami C, et al.; REGARD Trial Investigators. Ramucirumab monotherapy for previously treated advanced gastric or gastro-oesophageal junction adenocarcinoma (REGARD): an international, randomised, multicentre, placebo-controlled, phase 3 trial. Lancet. 2014 Jan;383(9911):31-9.

37 Wilke H, Muro K, Van Cutsem E, Oh SC, Bodoky G, Shimada Y, et al.; RAINBOW Study Group. Ramucirumab plus paclitaxel versus placebo plus paclitaxel in patients with previously treated advanced gastric or gastrooesophageal junction adenocarcinoma (RAINBOW): a double-blind, randomised phase 3 trial. Lancet Oncol. 2014 Oct;15(11):1224-35. 
38 Yen CJ, Muro K, Kim TW, Kudo M, Shih JY, Lee KW, et al. Ramucirumab Safety in East Asian Patients: A MetaAnalysis of Six Global, Randomized, Double-Blind, Placebo-Controlled, Phase III Clinical Trials. J Glob Oncol. 2018 Jul; 4(4):1-12.

39 Petrylak DP, de Wit R, Chi KN, Drakaki A, Sternberg CN, Nishiyama H, et al.; RANGE study investigators. Ramucirumab plus docetaxel versus placebo plus docetaxel in patients with locally advanced or metastatic urothelial carcinoma after platinum-based therapy (RANGE): a randomised, double-blind, phase 3 trial. Lancet. 2017 Nov;390(10109):2266-77.

40 Chau I, Park JO, Ryoo BY, Yen CJ, Poon R, Pastorelli D, et al. Alpha-fetoprotein kinetics in patients with hepatocellular carcinoma receiving ramucirumab or placebo: an analysis of the phase 3 REACH study. Br J Cancer. 2018 Jul;119(1):19-26.

41 Montal R, Andreu-Oller C, Bassaganyas L, Esteban-Fabró R, Moran S, Montironi C, et al. Molecular portrait of high alpha-fetoprotein in hepatocellular carcinoma: implications for biomarker-driven clinical trials. $\mathrm{Br} \mathrm{J}$ Cancer. 2019 Aug;121(4):340-3. 